\title{
NORMAS DE DISEÑO POR SISMO EN MÉXICO DF: ALGUNAS NOVEDADES INTERESANTES
}

\section{STANDARDS OF EARTHQUAKE DESIGN IN MEXICO CITY: SOME INTERESTING NEWS}

\section{MARIO ORDAZ}

Universidad Nacional Autónoma de México. México .malberto@algp.unam.mx

RESUMEN

La versión vigente de las Normas Técnicas Complementarias para Diseño por Sismo (NTCDS) del Reglamento de Construcciones del DF fue desarrollada entre 1999 y 2000, y fue promulgada en 2004. De entonces a la fecha se han llevado a cabo diversas investigaciones que, junto con ideas de cambio que han ocurrido en el mundo, han permitido desarrollar una nueva versión de las NTCDS que posiblemente entrarán en vigor en 2016.

En el presente trabajo se describen algunas de las modificaciones más novedosas, de entre las que destacan: a) una nueva "nanozonificación" sísmica que permite obtener espectros de diseño de sitio para casi todo el Distrito Federal; b) la inclusión de los cambios observados en las propiedades de los suelos por efecto del bombeo de agua; y c) los nuevos criterios para la especificación de sobreresistencia y reducciones por ductilidad. En adición, se discute la estructura general de la nueva norma y se reflexiona sobre las ventajas y limitaciones de las modificaciones propuestas.

PALABRAS CLAVE: códigos de diseño, nanozonación, sobre-resistencia, reducción de la ductilidad
The current version of the Complementary Technical Standards for Earthquake Design (NTCDS) of the Building Regulations of the DF was developed between 1999 and 2000, and was published in 2004. From then on, a number of investigations have been carried out which, together with Ideas of change that have occurred in the world, have allowed to develop a new version of the NTCDS that possibly will come into force in 2016.

In this paper, some of the most recent modifications are described, such as: a) a new seismic "nanozonation" that allows obtaining site design spectra for almost the entire Federal District; $b$ ) the inclusion of the changes observed in the properties of the soils due to the effect of water pumping; and c) the new criteria for the specification of over-resistance and reductions by ductility. In addition, it discusses the general structure of the new standard and reflects on the advantages and limitations of the proposed modifications.

KEYWORDS: design codes, nanozonation, over-resistance, ductility reduction 


\section{INTRODUCCIÓN}

Los avances logrados en los últimos 15 años en el estudio del comportamiento estructural y en la respuesta de los suelos de la Ciudad de México permiten que ahora se puedan plantear criterios de diseño más adecuados. En particular, en este trabajo se propone una manera de llegar a espectros de diseño de una manera más clara y acorde con el nuevo estado del conocimiento. Para esto, consideramos que deben satisfacerse al menos los siguientes requisitos (Miranda et al., 1999):

a) Los espectros elásticos deben representar de manera realista los verdaderos niveles de demanda que se presentarían ante los sismos de diseño. Esto incluye las restricciones impuestas por la dinámica estructural.

b) Las diferencias entre espectros elásticos de diseño en diferentes tipos de suelo deben reflejar correctamente los niveles de amplificación que se producen en la realidad.

c) Todas las reducciones a fuerzas o desplazamientos de diseño deben hacerse de manera explícita, aun cuando la adopción de los valores de reducción no pueda justificarse plenamente con bases teóricas o empíricas.

Con estos criterios generales se ha construido el procedimiento que se presenta a continuación.

\section{ESPECTROS ELÁSTICOS DE ACELERACIÓN}

Los espectros de diseño elástico son el punto de partida para el cálculo de las fuerzas laterales de diseño y para la determinación de las deformaciones laterales en las estructuras; de ahí su enorme importancia en el diseño sismorresistente. Es deseable, entonces, que ofrezcan al ingeniero indicaciones claras sobre los niveles de aceleración que pueden ocurrir en el sitio y sobre las máximas demandas, tanto de aceleración como de desplazamiento, que experimentarían las estructuras ahí desplantadas. Por otra parte, se reconoce que una manera razonable de especificar espectros de diseño es empezar con espectros de peligro sísmico uniforme, es decir, espectros cuyas ordenadas tienen la misma probabilidad de ser excedidas en un lapso dado.

En vista de lo anterior, el punto de partida para los espectros de diseño que aquí se proponen serán los espectros de peligro uniforme (seudoaceleración, 5\% del amortiguamiento crítico) calculados para distintos sitios de la Ciudad de México.

\section{ESPECTROS DE PELIGRO UNIFORME}

Para el cálculo de estos espectros, en primer lugar se calcularon, mediante procedimientos convencionales (Esteva, 1970), las curvas de tasa de excedencia de las ordenadas espectrales de interés (periodos entre 0 y 5 seg) para la Ciudad Universitaria (CU) de la Ciudad de México. Para esto se utilizaron las leyes de atenuación de Reyes (1999) desarrolladas específicamente para este sitio. Como se verá más adelante, CU es el sitio de referencia para el cálculo posterior de tasas de excedencia y espectros de peligro uniforme en otros sitios de la ciudad. Las tasas de excedencia calculadas han sido comparadas satisfactoriamente con las observadas, de acuerdo con lo reportado por Ordaz y Reyes (1999). En la figura 1 se presenta el espectro de peligro uniforme (EPU) para el sitio CU con periodo de retorno de 250 años. Conviene señalar que el espectro corresponde al promedio de las aceleraciones observadas en los componentes NS y EW. En la figura 1, junto con el EPU para la estación CU se presentan por separado los EPU que se tendrían si solo existieran eventos de subducción y si solo existieran eventos de profundidad intermedia. Más adelante quedará clara la razón de esta separación.

Nótese que este espectro de peligro uniforme incluye el efecto ponderado de sismos de diversos orígenes; el factor de ponderación está dado, principalmente, por la sismicidad de las diversas fuentes y su distancia a CU. En la figura 1 puede observarse que el espectro de peligro uniforme presenta dos máximos. El primero, en alrededor de $T=0.3 \mathrm{seg}$, se debe a los sismos locales y a los de profundidad intermedia que ocurren en la placa de Cocos subducida (ver, por ejemplo, Rosenblueth et. al, 1989); el segundo máximo, para $T=1 \mathrm{seg}$, está producido por los grandes temblores costeros.

Se obtuvieron, por otra parte, funciones de amplificación espectral, $F_{i}(T)$, para más de 100 sitios instrumentados en la Ciudad de México. Estas funciones, que dependen del periodo $T$, se definen como el promedio de los cocientes entre ordenadas del espectro de respuesta en el sitio de interés, digamos el i-ésimo, y el sitio CU, para el mismo periodo y diversos sismos:

$$
F_{i}(T)=\frac{1}{m i} \sum_{k=\tilde{\mathrm{n}}}^{m i} \frac{S_{\text {sok }}(T)}{S o o k}
$$

donde $m_{i}$ es el número de sismos registrados simultáneamente en la estación $i$ y en la estación $C U$, y $S a_{i k}(T)$ y $S a_{o k}(T)$ son las ordena- 
das espectrales registradas en la estación $i$ y en $C U$, respectivamente, durante el sismo $k$.

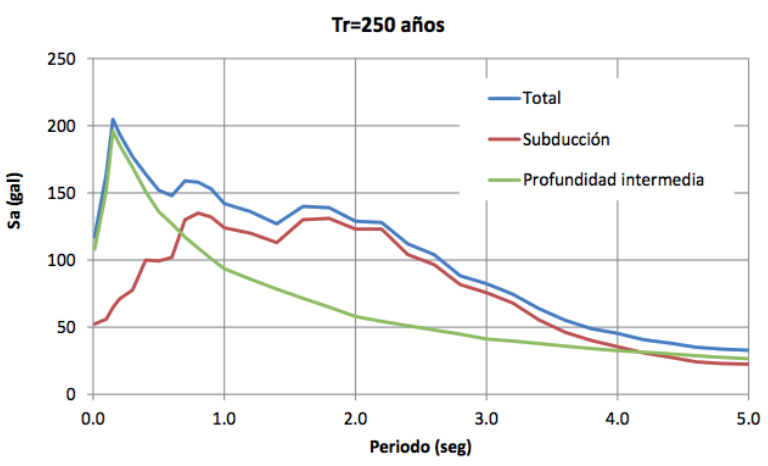

Figura 1. Espectros de peligro uniforme (250 años de periodo de retorno) para la estación CU.

Hecho lo anterior, se formó una malla de 1600 puntos cubriendo la porción más poblada del $\mathrm{DF}$, y se calculó para todos ellos la función de amplificación espectral $F_{j}(T), j=1,1600$, mediante el procedimiento de interpolación ideado por Pérez Rocha (1999). A partir de las tasas de excedencia en CU y las funciones de amplificación espectral es posible determinar el espectro de peligro uniforme para cada uno de los 1600 sitios, observando que (Esteva, 1970):

$$
V i[S a(T)]=V o\left[\frac{S a(T)}{F j(T)}\right]
$$

donde $v_{j}[S a(T)]$ es la tasa de excedencia de la aceleración $S a(T)$ en el sitio $j$, mientras que $v_{o}[S a(T)]$ es la tasa de excedencia de $S a(T)$ en la estación $C U$.

Para que la ecuación 2 sea estrictamente válida, deben cumplirse las condiciones siguientes: 1) que la función $F_{j}(T)$ sea constante de temblor a temblor; y 2) que la función $F_{j}(T)$ sea determinista, es decir, que no exista incertidumbre sobre su valor. Con rigor, ninguna de las dos condiciones se cumple. Hablando de la condición 1 , puede demostrarse que el cociente entre espectros de respuesta, aún con comportamiento lineal del suelo, depende del contenido de frecuencias del movimiento incidente. Adicionalmente, para niveles muy elevados de aceleración, es seguro que se presentaría comportamiento no lineal del suelo. Por otra parte, sí existe incertidumbre en la función $F_{j}(T)$ . Sin embargo, las aproximaciones implícitas en la ecuación 2 son aceptables en vista de que: 1) aunque $F_{j}(T)$ no es constante de temblor a temblor, tampoco varía mucho, ni siquiera cuando se trata de sismos de diversos orígenes (Singh et al, 1999); 2) los periodos de retorno que en este trabajo se recomiendan para diseño están asociados a sismos en que los efectos no lineales serán seguramente moderados o bajos, tal como ocurrió durante el sismo del 19 de septiembre de 1985; y 3) la incertidumbre en $F_{j}(T)$ es pequeña en comparación con la incertidumbre en las leyes de atenuación usadas en los cálculos de peligro (Pérez Rocha, 1999).

Conviene hacer notar que, como han demostrado Arroyo et al (2013), las características de los suelos blandos de la Ciudad de México han ido variando con el tiempo obedeciendo, principalmente, a la rigidización del suelo que ha acontecido como consecuencia de la extracción de agua. La figura 3 presenta un ejemplo de cómo ha ido variando el periodo predominante de vibrar con el transcurso del tiempo en una estación de la Ciudad de México.

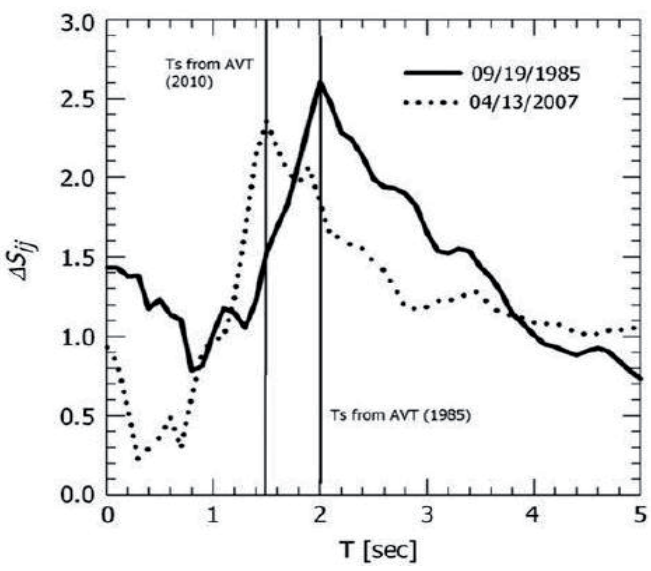

Figura 2. Variación de la función de amplificación, $F_{j}(T)$, para diferentes eventos sísmicos registrados en la estación SCT. Las líneas verticales son los periodos predominantes de vibrar obtenidos de pruebas de vibración ambiental. Tomada de Arroyo et al. (2013).

Esto quiere decir que, al menos para algunos sitios de la ciudad, es necesario tomar en cuenta que la función de amplificación a que se refiere la ecuación 1 debe cambiar con el tiempo. Para ello, se ha utilizado el modelo desarrollado por Arroyo et al (2013) para estimar los valores a que llegaría el periodo predominante de vibrar en diferentes momentos del futuro. En la figura 2 se muestran mapas de esta cantidad, para diferentes épocas. Puede observarse que, según este modelo predictivo, a largo plazo todo el terreno blando de la Ciudad de México será una gran zona de transición.

El cambio en el periodo predominante de vibrar lleva aparejado un cambio en la función de amplificación, tanto en lo referente a contenido de frecuencias como en lo que se refiere a amplificación. En la figura 3 se presenta un ejemplo de estos cambios para un sitio de la 
Ciudad de México. Luego, en la figura 4 se presentan los espectros de peligro uniforme que se calcularían, para cada época, multiplicando los EPU de terreno firme (figura 1) por las funciones de amplificación correspondientes. Nótese que, aunque la tendencia general de las amplificaciones es a disminuir, para un periodo estructural dado la peor situación puede no ser el presente sino algún punto en el futuro. Por ello, se ha optado por emplear para diseño la envolvente de los espectros entre 2010 y 2100.
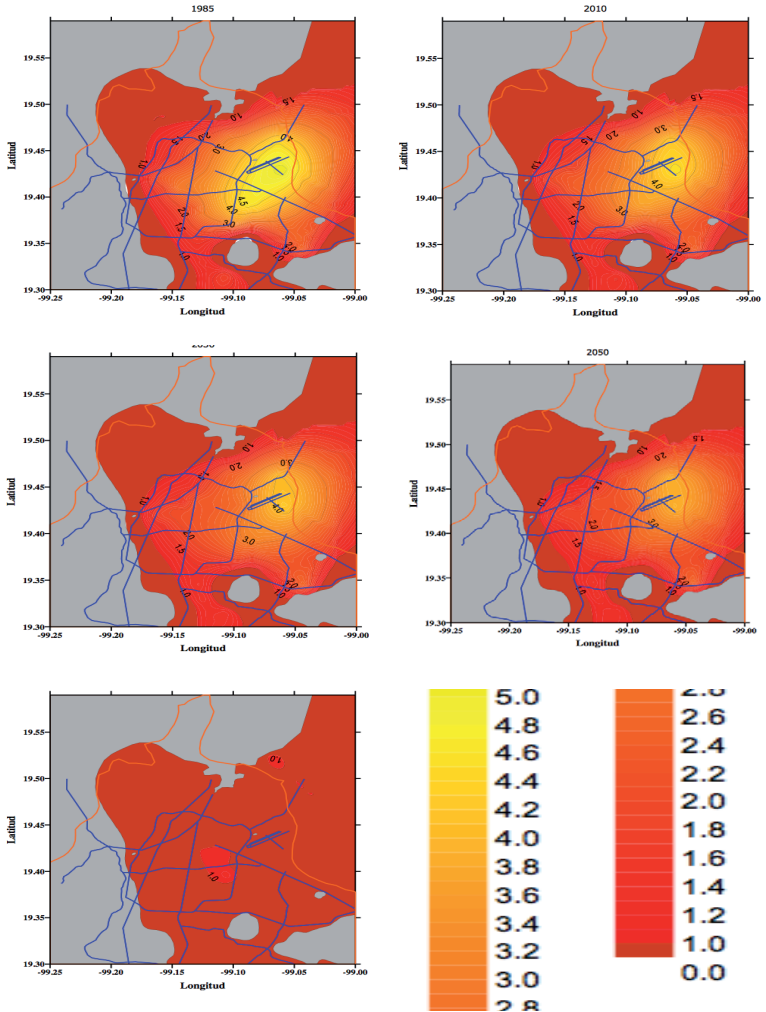

Figura 3. Evolución de los periodos predominantes de vibrar con el tiempo en la Ciudad de México, de acuerdo con el modelo de Arroyo et al (2013).

ESPECTROS DE PELIGRO UNIFORME SUAVIZADOS

Como puede observarse en la figura 4, los espectros de peligro uniforme tienen formas muy variadas, lo cual haría inconveniente su incorporación a las normas tal como están; es necesario entonces simplificar sus formas. Para ello, se ha elegido la siguiente forma paramétrica del espectro de aceleración, $S a(T)$ :

$$
\begin{aligned}
& \frac{S a(T)}{g}=\left\{\begin{array}{l}
a_{0}+\left(c-a_{0}\right) \frac{T}{T a} \\
c \\
c\left[k+(1-k)\left(\frac{T_{b}}{T}\right)^{2}\right]\left(\frac{T_{b}}{T}\right)^{2}
\end{array}\right. \\
& \text { si } T<T_{a} \\
& \text { si } T_{a} \leq T<T_{b} \\
& \text { si } T \geq T_{b}
\end{aligned}
$$

Se observa que la forma del espectro suavizado depende de 5 parámetros: $a_{o}$, que es la aceleración máxima del terreno; $c$, que es la ordenada espectral máxima; $T_{a}$ y $T_{b}$, que son periodos característicos del espectro; y $k$, que como se verá a continuación, es un parámetro que controla la caída de la ordenada espectral para $T>T_{b}$. Las formas espectrales resultantes pueden apreciarse en la figura 5 , junto con sus correspondientes espectros de desplazamiento, $S d(T)$.

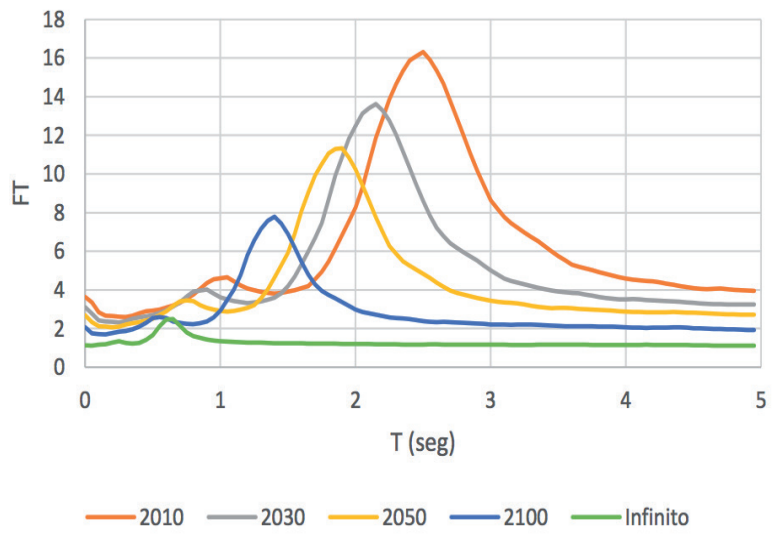

Figura 4. Evolución de las funciones de amplificación espectral con el tiempo para un sitio de la Ciudad de México.

Nótese que las formas espectrales para $T<T_{b}$ son las mismas que se han usado en los códigos mexicanos desde hace muchos años. Sin embargo, para $T>T_{b}$ se propone una forma nueva, con el propósito de tener una descripción más adecuada de los espectros de desplazamiento en ese intervalo de periodos.

A periodos largos el desplazamiento espectral tiende a una constante, que es el desplazamiento máximo del suelo, $D_{\max }$. En vista de la relación entre seudoaceleración y desplazamiento $\left(S d=S a T^{2} / 4 \pi^{2}\right)$; lo anterior sólo puede lograrse si el espectro de seudoaceleración decae al menos como $T^{2}$ para periodo suficientemente largo. Las formas que estipulan prácticamente todos los reglamentos del mundo para el espectro de seudoaceleración señalan un decaimiento más lento, lo cual conduce a que el espectro de desplazamiento, en muchos casos, crezca indefinidamente con el periodo. Esto es inadecuado, especialmente en sitios blandos como los que existen en la Ciudad de México, en donde pueden presentarse grandes desplazamientos espectrales para $T \approx T_{s}, \mathrm{y}$ desplazamientos considerablemente menores para $T \gg T_{s}$. Como ejemplo, el registro 
obtenido en la SCT durante el sismo del 19 de septiembre de 1985

(componente EW) presenta un desplazamiento espectral máximo de $120 \mathrm{~cm}$ pero un valor de $D_{\max }$ (que es también el espectral para $T>>T_{s}$ ) de sólo alrededor de $20 \mathrm{~cm}$.

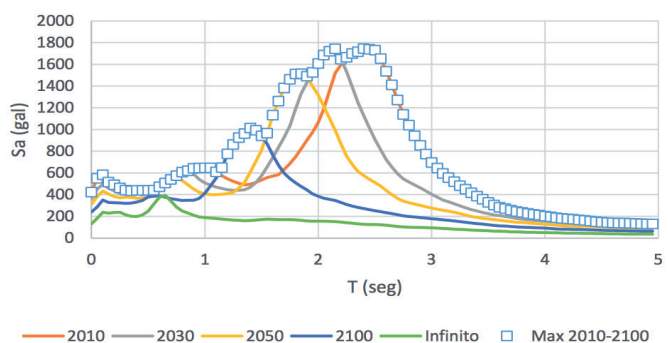

Figura 5. Evolución de los espectros de peligro uniforme (250 años) con el tiempo para un sitio de la Ciudad de México. La curva marcada como "MAX 2010-2100" es la envolvente de los espectros en el tiempo.
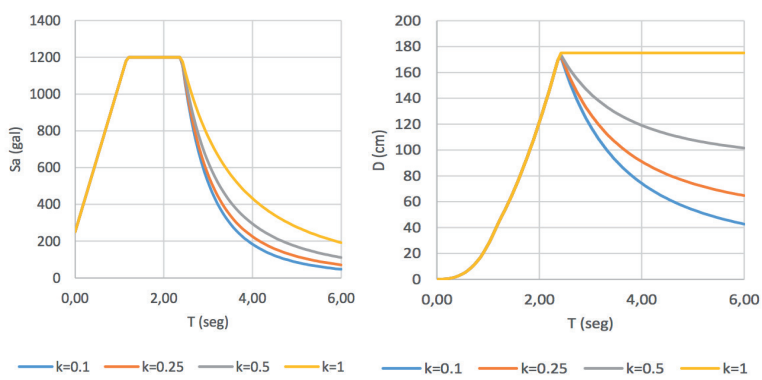

Figura 6. Izquierda: espectros de aceleración correspondientes a las formas analíticas dadas en la ecuación 3, para diferentes valores de $k$. Derecha, igual, pero para espectros de desplazamiento. Nótese la variación de las ordenadas para $T>T_{b}$ dependiendo del parámetro $k$.

En la figura 5, la forma espectral propuesta para $T>T_{b}$ conduce a espectros de desplazamiento más realistas, y es suficientemente rica como para representar tanto espectros de desplazamiento típicos de terreno firme $(k=1)$ como espectros de suelos muy blandos $(k=0)$.

Este nuevo parámetro $k$ tiene significado físico. En la ecuación 3 puede observarse que, independientemente del valor de $k$, cuando $T$ tiende a infinito, el espectro de seudoaceleración tiende a cero, pero el espectro de desplazamiento tiende a una constante $\left(D_{\max }\right)$ dada por:

$$
D_{\max }=\frac{c K T_{b}^{2}}{4 \pi^{2}}
$$

Por otro lado, el desplazamiento espectral máximo, que ocurre cuando $T=T_{b}$, vale

$$
S d_{\max }=\frac{c T_{b}^{2}}{4 \pi^{2}}
$$

de donde puede deducirse que el coeficiente $k$ es justamente el cociente entre el desplazamiento máximo del suelo y el desplazamiento espectral máximo:

$$
K=\frac{D_{\max }}{S d_{\max }}
$$

A partir de las formas espectrales seleccionadas (ecuación 3), se procedió a determinar, para cada uno de los 1600 puntos estudiados, los valores de los cinco parámetros del espectro suavizado, de manera que el espectro de peligro uniforme quedara cubierto en todo el intervalo de periodos.

\section{ESPECTROS ELÁSTICOS DE DISEÑO}

Determinados los parámetros del espectro suavizado para los 1600 punto estudiados, es posible fijar espectros de diseño para, virtualmente, cualquier punto de la Ciudad de México. Sin embargo, la variación espacial de los valores de los parámetros es más bien compleja, por lo que se decidió almacenar la información correspondiente en un sistema de cómputo que, a solicitud del usuario, proporciona el espectro de diseño para puntos arbitrarios en la Ciudad. En la figura 6 se presenta un ejemplo de la pantalla de este sistema.

En la parte derecha de la pantalla se presenta un mapa de la Ciudad de México, que sirve para seleccionar el sitio; la cantidad que se encuentra mapeada es el valor del parámetro c (ver ecuación 3) que es el máximo del espectro de aceleraciones. En la parte derecha se presentan diversos espectros: el de peligro uniforme para el sitio, el espectro elástico sin reducir (dado por la ecuación 3) y los espectros que resultan de aplicar diversas reducciones de las que se detalla más adelante.

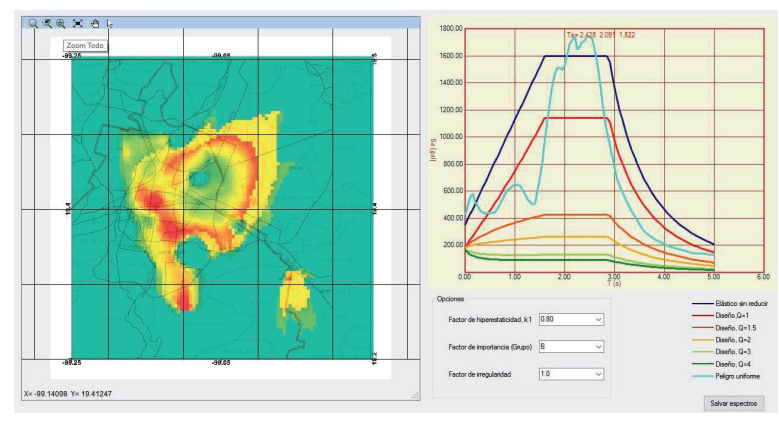

Figura 7. Pantalla del sistema de cómputo que proporciona los espectros de diseño para la Ciudad de México.

REDUCCIÓN POR DUCTILIDAD Los criterios contemporáneos de diseño admiten que la estructura tenga incursiones en el rango no lineal ante el sismo que caracteriza el estado límite de colapso. Esto permite limitar 
las demandas de fuerza en los elementos estructurales (para, por tanto, utilizar resistencias de diseño menores), a costa de que se presenten demandas de ductilidad limitadas y cierto nivel de daños provocados por la fluencia de algunas secciones de la estructura.

Para modelar el comportamiento no lineal, la mayoria de los reglamentos del mundo están basados en análisis de un sistema de un grado de libertad con comportamiento elastoplástico. Es con este modelo que se determina la resistencia necesaria para limitar las demandas de ductilidad a un valor especificado, que llamaremos $Q$. Es usual expresar la resistencia necesaria para lograr una demanda de ductilidad dada, $C(T, Q)$, como una fracción de la resistencia necesaria para tener una demanda de ductilidad unitaria (comportamiento elástico), $C(T, I)$ ; nótese que ambas resistencias dependen del periodo estructural, $T$. Llamaremos $Q^{\prime}$ a este cociente:

$$
Q^{\prime}(T, Q)=\frac{C(T, 1)}{C(T, Q)}
$$

La forma de la función $Q^{\prime}$ ha sido ampliamente estudiada en los últimos años (Krawinkler et al., 1992; Miranda, 1993; Miranda y Bertero, 1994). En particular, Ordaz y Pérez Rocha (1998) observaron que, bajo circunstancias muy generales, $Q^{\prime}$ depende del cociente entre el desplazamiento espectral, $S d(T)$ y el desplazamiento máximo del suelo, Dmax, de la siguiente manera:

$$
Q^{\prime}(T, Q)=1+(Q-1)\left(\frac{S d(T)}{C_{\max }}\right)^{\beta}
$$

donde $\beta=0.5$. Una versión simplificada de esta relación es la que se presenta a continuación:

$$
Q_{=}^{\prime}= \begin{cases}1+\frac{Q-1 t}{\sqrt{k}} \frac{T}{T_{a}} ; & \text { si } T \leq T_{a} \\ 1+\frac{Q-1 t}{\sqrt{k}} ; & \text { si } T_{a}<T \leq T_{b} \\ 1+(Q-1) \sqrt{\frac{P}{k}} ; & \text { si } T>T b\end{cases}
$$

donde

$$
p=k+(1-k)\left(\frac{T_{b}}{T}\right)^{2}
$$

Para llegar a esta expresión aproximada, se razonó de la siguiente manera: independientemente de $Q$, para $T=0, Q^{\prime}(T, Q)=1$. Por sencillez, se decidió hacer una variación lineal entre $Q^{\prime}=1$ para $T=0$ y $Q^{\prime}=Q_{\max }$ para $T=T_{a} . Q_{\max }$ es el máximo valor que puede llegar a tomar $Q^{\prime}$. De acuerdo con la expresión 13 , este máximo se presentará cuando $S d(T)$ sea máximo, lo cual acontece para $T=T_{b}$. En este periodo, de acuerdo con la ecuación 9, $S d(T) / D_{\max }=I / k$, de donde se desprende que, si $\beta=0.5$,

$$
Q_{\max }=1+\frac{Q-1}{\sqrt{k}}
$$

De aquí las expresiones de los dos primeros tramos de la ecuación 9. La expresión para $T>T_{b}$ , resulta de considerar que si en este intervalo el espectro de seudoaceleración sigue lo señalado en la ecuación 3, entonces su correspondiente espectro de desplazamiento será

$$
S d(T)=c\left(\frac{T_{b}}{2 \pi}\right)^{2}\left[k+(1-k)\left(\frac{T_{b}}{T}\right)^{2}\right]
$$

Sustituyendo las ecuaciones 4 y 17 en la ecuación $13 \operatorname{con} \beta=0.5$, se obtiene la expresión para $Q^{\prime}$ cuando $T>T_{b}$ que se presenta en la ecuación 14. Nótese que $Q^{\prime}$ depende tanto de $Q$ como de los parámetros $T_{a}, T_{b}$ y $k$. Estos, como se ha indicado, dependen del sitio. En la figura 7 se presentan ejemplos de la función $Q^{\prime}$ para $Q=4$ y diferentes valores de $k$.

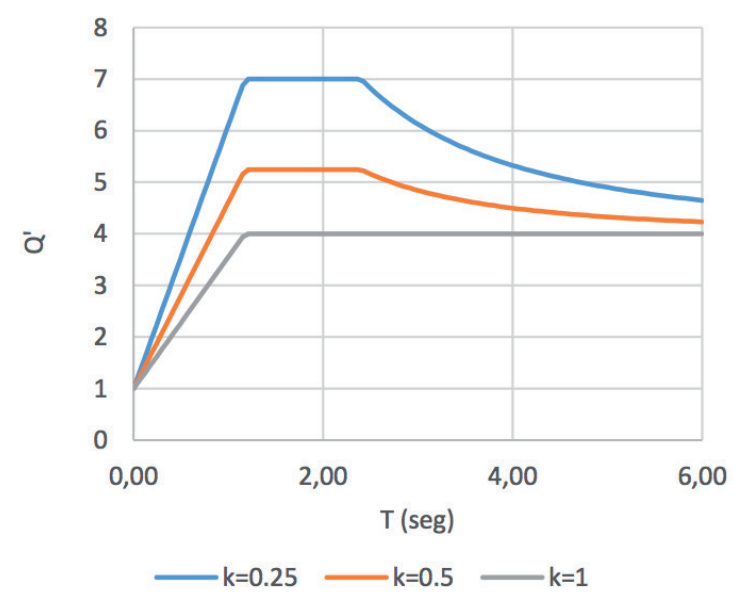

Figura 8. Valores de la reducción por ductilidad, $Q^{\prime}$, para $Q=4$ y diferentes valores de $k$.

Obsérvese que, a diferencia de lo que ocurre con la reducción por ductilidad en las normas vigentes, el valor de $Q^{\prime}$ puede ser más grande que $Q$. De acuerdo con la ecuación 11 , esto ocurre sólo cuando $K<1$, lo cual a su vez sucede solo para suelos blandos.

Que en suelos blandos $Q^{\prime}$ puede ser mayor que $Q^{\prime}$ en cierto intervalo de periodos fue notado por primera vez por Meli y Ávila (1986) analizando los registros obtenidos en zona de lago 
durante el sismo del 19 de septiembre de 1985. Este hecho se ha verificado posteriormente analizando cientos de acelerogramas (Miranda, 1993; Ordaz y Pérez Rocha, 1998). Para periodo muy largo $\left(T>T_{b}\right)$, puede verificarse que, como lo exige la dinámica estructural, $Q^{\prime}$ tiende a $Q$.

REDUCCIÓN POR SOBRERRESISTENCIA

La existencia de sobrerresistencia estructural ha sido reconocida ya en diversos reglamentos de construcción en el mundo (e.g., Rosenblueth et al., 1989). Existen diversas fuentes de sobrerresistencia. Se tiene, por un lado, que los valores nominales de las resistencias de los materiales son, por definición, generalmente menores que sus resistencias reales. Por otro lado, muchos de los modelos que se emplean para evaluar resistencias tienen simplificaciones del lado de la seguridad; puede consultarse el trabajo de Miranda (1991) para una discusión profunda de estos aspectos.

Pero quizá la fuente más grande de sobrerresistencia en muchas estructuras sea el procedimiento mismo de diseño que se utiliza en las disposiciones reglamentarias. Como se recuerda, las normas especifican que la estructura debe analizarse ante fuerzas reducidas por el factor $Q^{\prime}$, y debe suministrarse a las secciones una resistencia tal que se mantengan elásticas ante dichas fuerzas. Por tanto, si una sección fluye ante las cargas reducidas, deberá aumentarse su resistencia hasta que esto no ocurra. Se supone entonces que la resistencia nominal de la estructura es aquella con la cual ninguna sección fluye. En realidad algunas secciones fluirán ante el sismo de diseño, y los requisitos normativos están orientados a que las demandas de ductilidad en estas secciones no sobrepasen su capacidad. Sin embargo, el comportamiento global de la estructura no es, en general, estrictamente elastoplástico; sólo lo sería si todas las secciones fluyeran al mismo tiempo, lo cual puede ocurrir sólo en estructuras de pocos grados de libertad. Esto implica que la resistencia global real de la estructura es superior a la nominal.

Como ha observado Loera (2000), este efecto debería tomarse en cuenta cuando se evalúan las resistencias y no como un factor reductivo de las cargas; sin duda, este sería el procedimiento más racional. Esto implicaría, sin embargo, cambios profundos en los criterios de análisis estructural y llevaría, casi seguramente, a la obligatoriedad del uso de métodos inelásticos de análisis. La alternativa que parece más viable por el momento es el método del empujón. Aunque se ha avanzado en el estudio de estos métodos de análisis, es nuestra opinión que aún no se ha investigado lo suficiente como para modificar los esquemas actuales de análisis. En vista de lo anterior, se propone seguir aplicando la sobrerresistencia como un factor reductivo del lado de las acciones.

La sobrerresistencia depende de muchos factores; en particular, por lo que se asienta en párrafos anteriores, depende del grado de hiperestaticidad de la estructura. No se dispone de suficientes estudios que permitan calcular la sobrerresistencia en función de unos pocos parámetros estructurales. Por tal motivo, se propone utilizar factores reductivos que conduzcan, aproximadamente, a las resistencias que se obtienen con las normas actuales para valores de capacidad dúctil de entre 3 y 4 . Se propone entonces que la reducción por sobrerresistencia, R, esté dada por el factor

$$
R= \begin{cases}\frac{10}{4+\sqrt{\pi T / T_{a}}} & \text { si } T \leq T_{a} \\ 2 & \text { si } T>T_{a}\end{cases}
$$

$R$, como puede apreciarse, vale 2.5 para $T=0$ y 2 para $T>T_{a}$. No existen en realidad bases teóricas o empíricas para explicar una variación de $R$ como la que se presenta en la ecuación 13 . Se eligió esta forma funcional porque, como se ha señalado, conduce a resistencias necesarias del orden de las que se obtienen actualmente para $Q=3$ o 4 , y porque, como se verá más adelante, conduce a formas espectrales razonables.

\section{ESPECTROS INELÁSTICOS DE DISEÑO}

De acuerdo con lo señalado en los incisos anteriores, la resistencia necesaria de diseño, $C(T, Q)$, se calculará de la siguiente manera:

$$
C(T, Q)=\frac{a(T)}{R(T) Q^{\prime}(T, Q)}
$$

con $S a(T)$ dado por la ecuación $3, Q^{\prime}(T, Q)$ por la ecuación 9 y $R$ por la ecuación 14 . En la figura 6 se presentan espectros inelásticos de diseño para un sitio de la Ciudad de México y varios valores de ductilidad.

Para evitar estructuras con resistencias excesivamente bajas, se propone que, si del análisis se encuentra que la fuerza cortante $V$ es menor que $a_{\min } W$, donde $W$ es el peso de la estructu$\mathrm{ra}$, se incrementen todas las fuerzas de diseño en una proporción tal que $V$ iguale a ese valor; 
por las razones que se detallarán más adelante, los desplazamientos no deben afectarse por esta corrección. $a_{\min }$ se propone igual a 0.03 cuando $T_{a}<1 \mathrm{seg}$, o 0.05 cuando $T s>1 s$.

\section{CÁLCULO DE DESPLAZAMIENTOS}

Los desplazamientos se calcularán multiplicando por ciertos factores los que se obtienen de someter a la estructura a las fuerzas laterales reducidas, es decir, aquéllas que resultan de la distribución del cortante basal de diseño $C(T, Q) W$. Llamaremos a estos los desplazamientos reducidos, $D_{R}$. Se propone la verificación de los desplazamientos de la estructura en dos estados límite: el de colapso y el de servicio. Describiremos a continuación estos estados y la manera de calcular los desplazamientos en ambos casos.

ESTADO LÍMITE DE COLAPSO

Este estado límite intenta verificar el comportamiento -los desplazamientos relativos de entrepiso en particular- durante la ocurrencia del sismo de diseño, cuyo espectro elástico de aceleraciones queda descrito por la función $S a(T)$ (ver ecuación 3). Los desplazamientos ante este sismo, que llamaremos $D_{c}$, se calcularán, como es costumbre, multiplicando los reducidos, $D_{R}$, por $Q$. Pero, además, deberán multiplicarse por $R$, el factor de sobrerresistencia. La razón de esto es que, si en efecto la estructura tiene una sobrerresistencia $R$, las fuerzas sísmicas no quedarán limitadas por el valor de la resistencia de diseño, $C(T, Q)$, sino por una mayor, que es justamente el producto de la resistencia nominal por la sobrerresistencia. De acuerdo con esto,

$$
D_{c}=D_{r} Q R
$$

A partir de los desplazamientos de colapso $D_{c}$ , se calcularán las distorsiones de entrepiso resultantes, las cuales se compararán con distorsiones permisibles que reflejen la capacidad máxima real de los diversos sistemas estructurales. En la Tabla 1 se presentan algunos de los valores propuestos.

ESTADO LÍMITE DE SERVICIO

Se propone la existencia de un estado límite de servicio claramente especificado. Se pretende que la estructura permanezca elástica y con desplazamientos limitados ante la ocurrencia de un sismo que tiene un periodo de retorno mucho menor que el del sismo de colapso. El problema de la determinación del periodo de retorno óptimo del sismo de servicio no es trivial, y aunque se han hecho estudios al respecto (ver, por ejemplo, Reyes, 1999), el tema no está de ninguna manera agotado. Sin embargo, en el trabajo antes mencionado se señalan indicios de que, para diversos tipos estructurales comunes, el periodo de retorno óptimo para la Ciudad de México es de unos diez años. A este periodo de retorno están asociadas ordenadas espectrales similares a las que produjo el sismo del 25 de abril de 1989. Hay, además, un razonable acuerdo entre los expertos consultados en el sentido que, durante un sismo como este, no deberían presentarse daños no estructurales en las edificaciones.

Se propone por lo tanto, que el sismo de servicio sea uno con un espectro de aceleraciones como el de la ecuación 4 dividido entre un factor constante igual a 7, para tener espectros del orden de los registrados el 25 de abril de 1989. Se ha señalado en el pasado la inconveniencia de tener, en un esquema de diseño multinivel como el que aquí se propone, espectros de diseño que tengan la misma forma, especialmente en suelos blandos (Ordaz et al., 1996). La razón es que la forma espectral puede ser considerablemente modificada por el contenido de frecuencia de los sismos que rigen el estado límite de servicio, que no tienen las mismas características que los que rigen el estado límite de colapso. Sin embargo, en aras de la sencillez, se ha decidido dejar esta propuesta con una forma espectral igual para ambos estados límite. Esto, sin duda, ameritará ser afinado en el futuro.

En estas condiciones, los desplazamientos para revisión del estado límite de servicio, $D_{S}$, se calcularán de la siguiente manera:

$$
D_{s}=D_{r} \frac{Q^{\prime} R}{7}
$$

Al multiplicar los desplazamientos reducidos por $Q^{\prime}$ y por $R$ se tienen los desplazamientos elásticos que se presentarían durante el sismo de colapso; la posterior división entre 7 los convierte en los que se tendrían durante un sismo 7 veces menor. Las distorsiones de entrepiso resultantes de estos desplazamientos se compararán con valores de distorsión para los cuales se inicia el daño en sistemas no estructurales comunes. Se proponen los valores de 0.002 cuando los muros de mampostería estén ligados a la estructura y de 0.004 cuando no lo estén. 
TABLA 1. DISTORSIONES PROPUESTAS PERMITIDAS PARA EL ESTADO LÍMITE DE COLAPSO EN DIVERSOS SISTEMAS ESTRUCTURALES

\begin{tabular}{lc}
\hline SISTEMA ESTRUCTURAL & DISTORSIÓN \\
\hline Marcos dúctiles de concreto reforzado ( $\mathrm{Q}=3$ ó 4 ) & 0.0300 \\
\hline Marcos dúctiles de acero ( $\mathrm{Q}=3$ ó 4 ) & 0.0300 \\
\hline Marcos de acero con ductilidad limitada ( $\mathrm{Q}=1$ ó 2) & 0.0150 \\
\hline $\begin{array}{l}\text { Losas planas sin muros o contravientos } \\
\text { Marcos de acero con contravientos excéntricos }\end{array}$ & 0.0150 \\
\hline Marcos de acero o concreto con contravientos concéntricos & 0.0200 \\
\hline $\begin{array}{l}\text { Muros combinados con marcos dúctiles de concreto (Q=3) } \\
\text { Muros combinados con marcos de concreto con ductilidad }\end{array}$ & 0.0150 \\
\hline $\begin{array}{l}\text { limitada (Q=1 ó 2) } \\
\text { Muros diafragma }\end{array}$ & 0.0150 \\
\hline $\begin{array}{l}\text { Muros de carga de mampostería confinada de piezas macizas } \\
\text { con refuerzo horizontal o malla }\end{array}$ & 0.0100 \\
\hline $\begin{array}{l}\text { Muros de carga de: mampostería confinada de piezas macizas; } \\
\text { mampostería de piezas huecas confinada y reforzada horizontal- } \\
\text { mente; o mampostería de piezas huecas confinada y reforzada con } \\
\text { malla }\end{array}$ & 0.0050 \\
\hline $\begin{array}{l}\text { Muros de carga de mampostería de piezas huecas con } \\
\text { refuerzo interior }\end{array}$ & 0.0040 \\
\hline $\begin{array}{l}\text { Muros de carga de mampostería que no cumplan las especifica- } \\
\text { ciones para mampostería confinada ni para mampostería reforzada } \\
\text { interiormente }\end{array}$ & 0.0015 \\
\hline
\end{tabular}

DESPLAZAMIENTOS CUANDO GOBIERNA EL REQUISITO DE RESISTENCIA MÍNIMA

Como se ha señalado, cuando la resistencia calculada es menor que cierto valor, deben escalarse las fuerzas sísmicas resultantes de suerte que el coeficiente cortante basal sea justamente $a_{\min }$. Esta corrección, sin embargo, no debe afectar al cálculo de desplazamientos. Multiplicar los desplazamientos por este factor correctivo implica, aproximadamente, tener un espectro de diseño de aceleraciones que a partir de cierto periodo se vuelve constante. Con un espectro así, el espectro de desplazamiento empezaría a crecer como $T^{2}$ justamente a partir de ese periodo, lo que constituye una forma poco realista para dicho espectro.

ALGUNAS IMPLICACIONES DE LA FORMA PROPUESTA PARA LOS ESPECTROS DE DESPLAZAMIENTO

Se han observado por lo menos dos consecuencias importantes producidas por las nuevas formas del espectro de desplazamiento. Se mencionan brevemente a continuación.

1. En la figura 8 se presentan espectros de diseño de desplazamiento (estado límite de colapso) para un sitio con de la Ciudad de México. Puede observarse que el desplazamiento espectral alcanza un máximo (en este caso en $T=2.4 \mathrm{seg}$ ) y después empieza a disminuir, tendiendo, como dicta la dinámica estructural, al desplazamiento máximo del suelo. Esto significa que habrá casos en que el desplazamiento inelástico espectral necesario para producir la distorsión máxima de entrepiso será mayor que el máximo del espectro de diseño de desplazamiento. En esta situación, el estado límite de colapso no podrá gobernar en lo referente a rigidez de la estructura.

2. Como puede observarse de la figura 8, los desplazamientos de diseño a periodo corto e intermedio dependen de la resistencia de la estructura y, por tanto, de la capacidad dúctil de diseño, $Q$. Esto contrasta con las normas convencionales en que, como consecuencia de que $Q^{\prime}=Q$, para periodos intermedios y largos el desplazamiento es independiente de la resistencia. Esto podría implicar un mayor número de iteraciones durante el proceso de diseño.

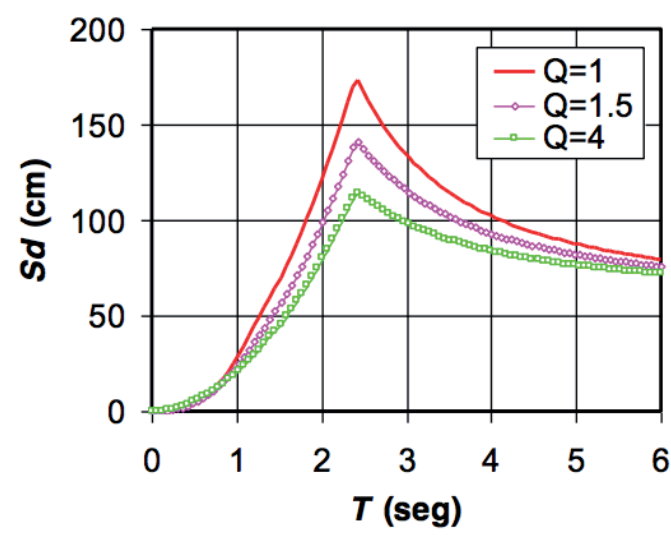

Figura 9. Desplazamientos de diseño (estado límite de colapso) para un sitio con de la Ciudad de México para diversos valores de ductilidad, $Q$.

\section{CONCLUSIONES}

Se ha presentado un procedimiento que permite determinar resistencias y desplazamientos de diseño en la Ciudad de México de una manera más clara, y más acorde con el estado actual tanto del conocimiento sobre respuesta de suelos blandos, cuanto de las tendencias modernas de los reglamentos de construcción. Las modificaciones más importantes que se proponen son las siguientes: 
- Se parte de espectros elásticos de aceleración y desplazamiento que tienen tamaños y formas realistas.

- Se aplica explícitamente una reducción empírica por sobrerresistencia.

- Se utilizan reducciones por ductilidad con reglas más acordes con las reducciones que se observan en la realidad para sistemas de un grado de libertad.

\section{REFERENCIAS BIBLIOGRÁFICAS}

Arroyo, D., Ordaz, M., Ovando, E., Guasch, J.C., Lermo, J., Pérez, C., Alcántara, L., Ramírez, M. (2013). "Evaluation of the change in dominant periods in the lake-bed zone of Mexico City produced by ground subsidence through the use of site amplification factors", Soil Dynamics and Earthquake Engineering, 44, 54-66.

Esteva, L. (1970), "Regionalización sísmica de México para fines de ingeniería”, Serie Azul 246, Instituto de Ingeniería, UNAM.

Krawinkler, H. y M. Rahnama (1992), "Effects of soils on design spectra", Proceedings 10th World Conference on Earthquake Engineering, Madrid, Spain, $10,5841-5846$.

Loera, S. (2000). Comunicación personal.

Meli, R. y J. Ávila (1988), "Analysis of building response", Earthquake Spectra 5, 1-18.

Miranda, E. (1993), "Site-dependent strength reduction factors", Journal of Structural Engineering, ASCE, 119, 3503- 3519.

Miranda, E. y V. Bertero(1994), "Evaluation of strength reduction factors for earthquake-resistant design", Earthquake Spectra 10, 357-379.

Miranda, E. (1999), "Approximate seismic lateral deformation demand in multistory buildings", Journal of Structural Engineering 125, 417-425.

Miranda, E., M. Ordaz y E. Reinoso (1999), "Algunas consideraciones sobre los nuevos reglamentos
- Se estipulan procedimientos más racionales para el cálculo de desplazamientos.

- Se hace explícita la existencia de dos estados límite de desplazamiento, con distorsiones de entrepiso permisibles que reflejan mejor el desempeño estructural que se quiere obtener.

mexicanos de construcción de diseño por sismo", XII Congreso Nacional de Ingeniería Sísmica. II. 1061-1070 Morelia, México.

Ordaz, M, E Reinoso y LE Pérez Rocha (1996), "Criterios de diseño sísmico: consideraciones para suelos blandos", Ingeniería Sísmica 53, 25-35.

Ordaz, M. y Pérez Rocha, L.E. (1998), "Estimation of strength-reduction factors for elastoplastic systems: a new approach”, Earthquake Engineering and Structural Dynamics 27 889-901.

Ordaz, M. y C. Reyes (1999), "Seismic Hazard in Mexico City: observations vs. computations", Bulletin of the Seismological Society of America 89, 1379-1383.

Pérez Rocha, L. E. (1999), "Respuesta sísmica estructural: efectos de sitio e interacción suelo estructura", Tesis Doctoral, Facultad de Ingeniería, UNAM, México.

Reyes, C. (1999), "El estado límite de servicio en el diseño sísmico de edificios", Tesis Doctoral, Facultad de Ingeniería, UNAM, México.

Rosenblueth E., M. Ordaz, F.J. Sánchez-Sesma, y S.K. Singh (1989), "Design Spectra for Mexico's Federal District”, Earthquake Spectra 5, 273291.

Singh, S.K., M. Ordaz, J Pacheco, R. Quaas, et. al (1999), "A preliminary report on the Tehuacán, Mexico, earthquake of June 15, 1999, Mw=7", Seismological Research Letters 70 489-504. 